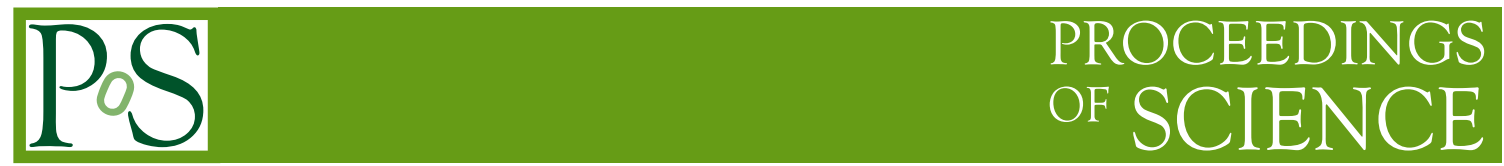

\title{
Interplay between the LHC and flavor physics
}

\section{Yuji Omura*}

Department of Physics, Kindai University, Higashi-Osaka, Osaka 577-8502, Japan

E-mail: yomuraephys.kindai.ac.jp

There are discrepancies between the experimental results and the Standard Model predictions, in the lepton flavor universality of the semileptonic $B$ decays: $B \rightarrow D^{(*)} \ell v$ and $B \rightarrow K^{(*)} l l$. In this paper, we discuss several new physics interpretations motivated by the excesses and study the signals of the new physics in the LHC and the dark matter experiments.

The 4th KMI International Symposium (KMI2019)

18-20, February 2019

Nagoya, Japan

${ }^{*}$ Speaker. 


\section{Introduction}

Recently, we can find discrepancies between the experimental results and the Standard Model (SM) predictions in the semileptonic $B$ decays. The $\mathrm{LHCb}$ collaboration has reported that the experimental results are deviated from the SM predictions in the $b \rightarrow$ sll processes. In the experiment, the branching fractions of $B \rightarrow K^{(*)} l l(l=e, \mu)$ are measured, and lepton flavor universalities (LFUs) and angular distributions are studied in each process. One excess is reported in the ratio between $\mathrm{BR}\left(B^{+} \rightarrow K^{+} \mu \mu\right)$ and $\mathrm{BR}\left(B^{+} \rightarrow K^{+} e e\right)$ in the region with $1 \mathrm{GeV}^{2} \leq q^{2} \leq 6 \mathrm{GeV}^{2}$, where $q^{2}$ is the invariant mass of two lepton in the final state [1]. The experimental result suggests the smaller value of $\operatorname{BR}\left(B^{+} \rightarrow K^{+} \mu \mu\right)$ than the SM prediction, and the deviation is about $2.6 \sigma$ [1]. Recently, a similar deviation is discovered in $B \rightarrow K^{*} \mu \mu$ [2]. The $B$ decay to $\mu$ pair in this process is again smaller than the SM prediction. Similar indications are also reported in $B \rightarrow \phi \mu \mu$ [3] and $\Lambda_{b} \rightarrow \Lambda \mu \mu$ [4] in the same $q^{2}$ region. Moreover, the disagreement between the experimental results and the SM prediction of the angular distribution in $B \rightarrow K^{*} \mu \mu$ is also one of the longstanding issues $[5,6]$. The CMS collaboration has shown the result that may be consistent with the SM prediction, but the deviation is still large in the LHCb experiment and the other. Thus, there might be some issues in the $b \rightarrow s$ transition associated with $\mu$.

In 2012, the BaBar collaboration has reported that there are large discrepancies in the LFUs of the semileptonic $B$ decays: $B \rightarrow D \ell v$ and $B \rightarrow D^{*} \ell v(\ell=e, \mu, \tau)$. The observables to measure the LFUs are defined as

$$
R\left(D^{(*)}\right)=\operatorname{Br}\left(B \rightarrow D^{(*)} \tau v\right) / \operatorname{Br}\left(B \rightarrow D^{(*)} l v\right)(l=e, \mu),
$$

and the experimental results are $R(D)=0.440 \pm 0.072$ and $R\left(D^{*}\right)=0.332 \pm 0.030[7,8]$. They are larger than the SM predictions. The $B$ decays associated with the light leptons are measured with good accuracy, so that the branching ratios of $B \rightarrow D^{(*)} \tau v$ are larger than the SM predictions. Interestingly, the Belle collaboration has also reported the excesses in $R\left(D^{(*)}\right)[9,10,11]$, although the discrepancies are milder than the BaBar results. Thus, it is expected that those excesses are the new physics signals and there are new particles that couple to the SM fermions flavor-dependently. We note that the LHCb collaboration has also reported only $R\left(D^{*}\right)$ and the latest result is consistent with the SM prediction at the $1 \sigma$ level $[12,13]$. Recently, the Belle experiment also reported the data of the $D^{*}$ polarization in the $B \rightarrow D^{*} \tau \nu$ process, and the result is slightly deviated from the SM prediction at $1.5 \sigma$ level [14].

Based on these deviations, a lot of new physics interpretations have been proposed. In this paper, we introduce some representative new physics explanations, and discuss how to test them in the collider experiments and the dark matter (DM) physics, based on Refs. [15, 16, 17, 18]. In particular, we see that the direct search for the new physics at the LHC plays an important role in testing them, since the excesses require sizable couplings with the SM $[15,18]$.

\section{The new physics interpretation of the $b \rightarrow s l l$ anomaly}

The LHCb collaboration has reported that there are deviations from the SM predictions in the $b \rightarrow$ sll processes. In those processes, namely $C_{7}, C_{9}$ and $C_{10}$ operators are relevant in the SM predictions. $C_{7}$ can not give sizable contributions to the processes, since it corresponds to 
the electric dipole operator that is strictly constrained by $B \rightarrow X_{s} \gamma$. In the region with $1 \mathrm{GeV}^{2}$ $\leq q^{2} \leq 6 \mathrm{GeV}^{2}$, where the excesses are reported by the $\mathrm{LHCb}$ collabration, the $C_{9}$ and $C_{10}$ operators dominantly contribute to the branching ratios. The Wilson coefficients, $C_{9}^{l}$ and $C_{10}^{l}$, are defined as follows:

$$
\frac{4 G_{F}}{\sqrt{2}} V_{t b} V_{t s}^{*} \frac{e^{2}}{16 \pi^{2}}\left\{C_{9}^{l}\left(\overline{s_{L}} \gamma_{\mu} b_{L}\right)\left(\bar{l} \gamma^{\mu} l\right)+C_{10}^{l}\left(\overline{s_{L}} \gamma_{\mu} b_{L}\right)\left(\bar{l} \gamma^{\mu} \gamma_{5} l\right)+\text { h.c. }\right\}
$$

In the SM, $C_{9}^{l}$ and $C_{10}^{l}$ are almost flavor universal and the sizes are estimated as about 4 , but the excesses seem to require sizable contributions to $C_{9}^{l}$ and $C_{10}^{l}$. ${ }^{*}$ In our study, $\Delta C_{9,10}^{l}$ denote the new physics contributions to $C_{9,10}^{l}$, respectively.

Many new physics scenarios have been proposed in order to explain the $b \rightarrow$ sll anomaly. One simple way is to introduce an extra gauged flavor symmetry. ${ }^{\dagger}$ In such a model, the non-vanishing charges are assigned to both $\mu$ and quarks and the extra gauge boson contributes to $\Delta C_{9,10}^{l}$ at the tree level. Although the $B_{s}$ meson mixing strongly constrains the contributions, the large $\Delta C_{9,10}^{l}$ can be achieved successfully if the charge of $\mu$ is not vanishing in the mass base. Another candidate for these excesses is namely leptoquark that is a scalar or vector field charged under $S U(3)_{c} .^{\ddagger}$ The new field couples both leptons and quarks, so that the tree-level exchanging can generate $\Delta C_{9,10}^{l}$.

We can discuss the other possibility that the large $\Delta C_{9,10}^{l}$ is realized by the one-loop diagrams involving extra fields [15]. For instance, extra vector-like quarks and leptons can be introduced without suffering from gauge anomaly. Further, Yukawa couplings between the SM fermions and the extra fields can be written down by introducing extra scalar fields. It is very interesting that we can consider the possibility that the extra field become a good DM candidate [15]. As pointed out in Ref. [21], however, this kind of model faces the stringent constraint from the direct-detection experiments of DM. The cross sections are almost on the upper bound of the latest LUX and XENON1T experiments $[22,23,24]$ if the observed DM relic density is explained by the thermal process. The scattering processes are induced by the photon and Z-boson exchanging at the oneloop level, and the contributions cancel each other in some parameter region. In addition, the tree-level diagrams involving the extra fermions become sizable depending on the alignment of the Yukawa couplings. Moreover, the direct search for extra quarks and leptons at the LHC is well developed recently. Therefore, the careful integrated study is required to discuss the excesses at the $\mathrm{LHCb}$ in this kind of model.

\subsection{Interplay between DM physics and flavor physics}

\subsubsection{Setup}

First of all, we introduce our model motivated by the $b \rightarrow s l l$ anomalies at the LHCb experiment, based on Ref. [15]. We introduce an extra SU(2) $)_{L}$-doublet quark, denoted by $Q^{\prime}$, and an extra

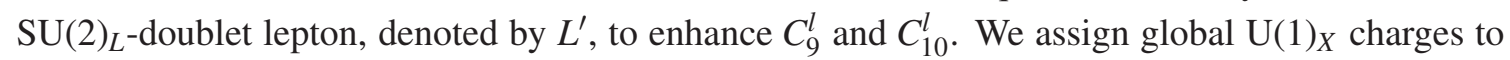
$Q^{\prime}$ and $L^{\prime}$ to distinguish them from the SM quarks and leptons. Note that all of the SM particles are neutral under the $\mathrm{U}(1)_{X}$. We also introduce a complex scalar $X$ as a candidate for DM. The DM $X$

\footnotetext{
${ }^{*}$ See, for example, Ref. [19].

${ }^{\dagger}$ See, for example, Ref. [16].

${ }^{\ddagger}$ See, for example, Ref. [20].
} 
is charged under the global $\mathrm{U}(1)_{X}$ symmetry, and it becomes stable if $X$ is lighter than both $Q^{\prime}$ and $L^{\prime}$. Note that $X$ is neutral under the SM gauge symmetry.

We can write down the potential for the fermions and the scalar, as shown in Ref. [15]. The relevant term is

$$
\lambda_{i}^{q} \bar{Q}_{R}^{\prime} X^{\dagger} Q_{L}^{i}+\lambda_{i}^{l} \overline{L_{R}^{\prime}} X^{\dagger} l_{L}^{i}+\text { h.c. }
$$

$\lambda_{i}^{q}$ and $\lambda_{i}^{l}$ is the Yukawa coupling between the DM and the extra fermions. The SM down-type quarks in $Q^{i}$ and the charged leptons in $l_{L}^{i}$ are the mass eigenstates, then we simply denote the Yukawa couplings as $\left(\lambda_{1}^{q}, \lambda_{2}^{q}, \lambda_{3}^{q}\right) \equiv\left(\lambda_{d}, \lambda_{s}, \lambda_{b}\right)$ and $\left(\lambda_{1}^{l}, \lambda_{2}^{l}, \lambda_{3}^{l}\right) \equiv\left(\lambda_{e}, \lambda_{\mu}, \lambda_{\tau}\right)$.

\subsubsection{The $b \rightarrow s l l$ anomaly with the observed DM abundance}

We can calculate the relic density and the cross section with nuclei, and find the correlation between the DM observables and the semileptonic $B$ decay. In the Fig. 1, we depict the values of $\Delta C_{9}$ by black lines, when $\left|\lambda_{\mu}\right|$ is aligned to account for the measured DM density. We assume $\left|\lambda_{s} \lambda_{b}\right|=0.15$, that corresponds to the upper bound from the $B_{s}-\overline{B_{s}}$ mixing [15], and consider two cases: $\left(\lambda_{b}, \lambda_{s}\right)=(1.0,0.15)$ (case A) and $\lambda_{b}=\lambda_{s}=\sqrt{0.15}$ (case B). In both cases, $\lambda_{d}$ is assumed to be negligibly small. The left (right) panel corresponds to the case A (case B). Shaded region is excluded by the LHC, flavor and DM experiments; the extra quark and lepton searches at the LHC (blue), the $B_{s}-\overline{B_{s}}$ mixing observables (green) and the DM direct detections (red). We also fill in the region constrained by the theoretical requirement with colors; the perturbative bound $\left|\lambda_{\mu}\right|>\sqrt{4 \pi}$ (gray) and the unstable $X, m_{X}>m_{L^{\prime}}$ (light gray).

In the case (A) (left panel), we see that the $Q^{\prime}$ searches at the LHC and the DM direct detection stringently limit our model parameter. Then, the region where $\Delta C_{9}^{\mu} \lesssim-0.2$ is excluded by these results. Following Ref. [19], the global fitting of $\Delta C_{9}^{\mu}=-\Delta C_{10}^{\mu}$ suggests $-0.81 \leq \Delta C_{9}^{\mu} \leq-0.48$ $(1 \sigma)$ and $-1.00 \leq \Delta C_{9}^{\mu} \leq-0.32(2 \sigma)$. Thus, we conclude that it is difficult to explain the $b \rightarrow$ sll anomalies within even $2 \sigma$ in the case (A). In the case (B), on the other hand, we find that even the region where $\Delta C_{9}^{\mu} \lesssim-0.3$ is allowed and the $b \rightarrow$ sll anomalies can be explained within $2 \sigma$, consistently with the other experiments.

\section{The new physics interpretation of the $b \rightarrow c l v$ anomaly}

There are large discrepancies in the LFUs of the semileptonic $B$ decays: $B \rightarrow D \ell v$ and $B \rightarrow$ $D^{*} \ell v(\ell=e, \mu, \tau)$. Motivated by the excesses, several new physics interpretations have been proposed. One simple way to violate the LFU in the $B$ decay is to introduce a field that couples to $\tau$ lepton. The field needs to couple to the heavy quarks, bottom and charm quarks, as well. One good candidate for such a field is a charged scalar, $H_{ \pm}$, that has Yukawa couplings with the heavy quarks and heavy leptons [17]. The Yukawa couplings are, in general, flavor-dependent, so that we can assume that the couplings with bottom, charm and $\tau$ lepton are relatively large, compared to the other elements. Then, the exchange of a charged scalar at the tree-level induces the violation of the LFU.

Instead of the charged scalar, we can discuss a charged vector, $W_{ \pm}^{\prime}$, that dominantly couples to the second and third generations [18]. In order to introduce such a vector field, additional gauge symmetry is required and the SM gauge symmetry may be extended. In addition, a non-trivial setup 

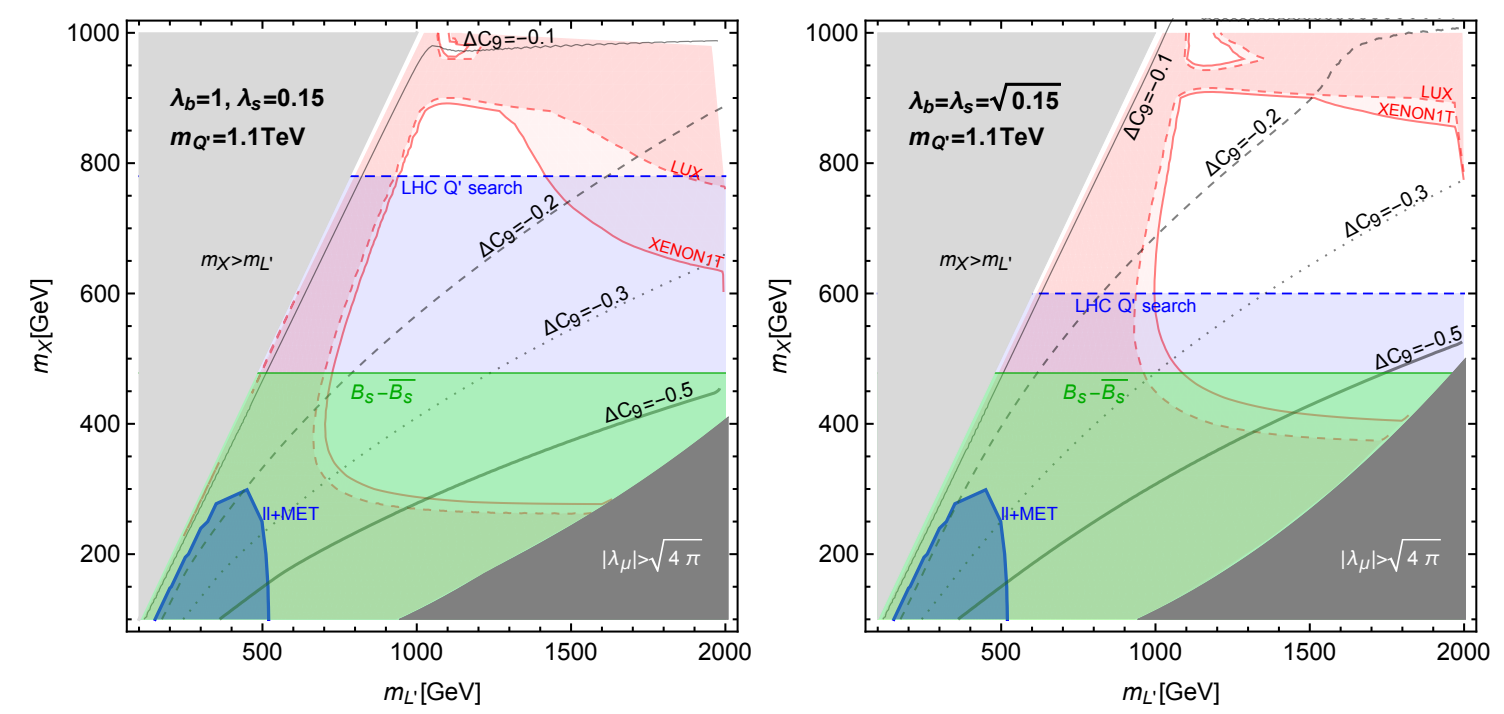

Figure 1: The $\Delta C_{9}^{\mu}$ value (black lines), when the value of $\left|\lambda_{\mu}\right|$ is aligned to explain the observed DM abundance [15]. The left (right) panel corresponds to the case A (case B). We fill in the region excluded by the LHC, flavor and DM experiments with colors; the extra quark and lepton searches (blue), $B_{s}-\overline{B_{s}}$ mixing (green) and DM direct detection experiments (red). The gray region stands for the perturbativity limit; $\left|\lambda_{\mu}\right|>\sqrt{4 \pi}$.

would be necessary to make the $W_{ \pm}^{\prime}$ couplings flavor-dependent. For instance, we can discuss a gauged flavor symmetry or we can expect that some heavy fermions effectively induce the flavorful couplings according to the mass mixing with the SM fermions.

Below, we focus on those two new physics interpretations and discuss the consistency with the direct search for the new phenomena at the LHC in the each setup. We study the $\tau v$ resonance search at the LHC.

\subsection{The explanation of the $R\left(D^{(*)}\right)$ anomaly}

In the semileptonic $B$ decays, $B \rightarrow D^{(*)} \ell v$, the SM predictions are given by the tree-level diagrams. Then, relatively large new interaction is required to compensate the SM contribution. If there is a heavy charged particle that couples to quarks and leptons flavor-dependently, the following operators could be generated by the heavy particle exchange:

$$
\begin{aligned}
\mathscr{H}_{e f f}= & \left(C_{S M}^{V}+C_{L}^{V}\right)\left(\overline{b_{L}} \gamma_{\mu} c_{L}\right)\left(\overline{v_{\tau L}} \gamma^{\mu} \tau_{L}\right)+C_{R}^{V}\left(\overline{b_{R}} \gamma_{\mu} c_{R}\right)\left(\overline{v_{\tau R}} \gamma^{\mu} \tau_{R}\right) \\
& +C_{L}^{S}\left(\overline{b_{R}} c_{L}\right)\left(\overline{v_{\tau L}} \tau_{R}\right)+C_{R}^{S}\left(\overline{b_{L}} c_{R}\right)\left(\overline{v_{\tau L}} \tau_{R}\right)+h . c . .
\end{aligned}
$$

$C_{S M}^{V}$ expresses a SM contribution generated by $W$ boson, with $C_{S M}^{V}=4 G_{F} V_{c b}^{*} / \sqrt{2}$. The two terms in the fist line can be generated by the $W^{\prime}$ exchange. In the extended SM with extra non-abelian gauge symmetry, massive extra gauge bosons are predicted. If the SM quarks are charged under the extra gauge symmetry, an extra charged gauge boson, $W^{\prime}$, may couple to the third-generation quark and lepton as

$$
\mathscr{L}_{W_{I}^{\prime}}=W_{I \mu}^{\prime}\left\{g_{I}\left(\overline{b_{I}} \gamma^{\mu} c_{I}\right)+g_{I \tau}\left(\overline{\tau_{I}} \gamma^{\mu} v_{\tau I}\right)\right\}+\text { h.c. },
$$


where, $I$ denotes the chirality: $I=L, R$. The couplings $g_{I}$ and $g_{I \tau}$ depend on the detail of the setup, and the other couplings involving light SM fermions may arise at the low energy. Assuming the third-generation couplings are dominant and $W^{\prime}$ is enough heavy, we can expect that $C_{L}^{V}$ and $C_{R}^{V}$ are effectively generated.

The last two terms can be from the $H_{ \pm}$exchange. The charged scalar can be introduced by adding extra Higgs $\mathrm{SU}(2)_{L}$ doublets. The Yukawa couplings between $H_{ \pm}$and the SM fermions depend on the setup, but in general the scalar couples to all of the SM fermions. Assuming a specific parameter choice, we can focus on the $b \rightarrow c$ transition induced by the Yukawa coupling of charged scalar, i.e.,

$$
\mathscr{L}_{H_{ \pm}}=-H_{-}\left\{Y_{R}\left(\overline{b_{R}} c_{L}\right)+Y_{L}\left(\overline{b_{L}} c_{R}\right)+Y_{\tau}\left(\overline{\tau_{R}} v_{\tau L}\right)\right\}+\text { h.c.. }
$$

Integrating out $H_{ \pm}$, we obtain $C_{L}^{S}$ and $C_{R}^{S}$ effectively.

\subsection{Interplay between the flavor physics and the LHC}

We study the signal of the each scenario at the LHC in the scenarios mentioned above. In our models, the charged resonances $\left(V^{+}=W_{I}^{\prime+}, H_{+}\right)$are produced in association with the thirdgeneration quark and decay to $\tau \nu$ and $b c$ as $g c \rightarrow V^{+} b \rightarrow \tau^{+} \nu b$.

The search for heavy $\tau v$ resonances has been performed at the LHC and severely constrain various models. We numerically found that the analysis reported by the CMS collaboration using the data at the LHC Run II with $35.9 \mathrm{fb}^{-1}$ [25] sets the most stringent bound on our models, where they focus on the $W^{\prime}$ heavier than $400 \mathrm{GeV}$ with the universal couplings to quarks of all generations.

Since the heavy resonances are only couples to the third generation in our models and the spin structures of the $H_{ \pm}$and the $W^{\prime}$ are different, the efficiency and the acceptance of the selection cut should be estimated by the simulation. The detail of our analysis is shown in Ref. [18].

We show the resulting $95 \%$ CL upper bound on the signal cross section times its branching ratio to $\tau v$ mode as a function of the resonance mass $m_{V}$ for each model in Figure 2. The difference of the spin structure provides different upper bounds, for charged scalar (red thick solid), $W_{L}^{\prime}$ (blue thick solid), and $W_{R}^{\prime}$ (green thick solid). The constraint on the charged scalar case is more stringent than the other cases in most of the $m_{V}$ region because of the harder $m_{T}$ distribution.

We also overlay the expected signal cross sections in the same plot for the three cases, in the red hatched region $\left(H_{ \pm}\right)$, in the blue hatched region $\left(W_{L}^{\prime}\right)$ and in the green hatched region $\left(W_{R}^{\prime}\right)$, assuming the couplings are compatible to accommodate the $R\left(D^{(*)}\right)$ observation in $1 \sigma$ level. As shown in Fig. 2, we conclude that the charged Higgs scenario is almost excluded unless the charged Higgs is lighter than $400 \mathrm{GeV}$. The $W^{\prime}$ case is also excluded, if $W^{\prime}$ couples to only right-handed fields.

\section{Summary}

In this paper, we have discussed the new physics interpretations of the excesses in the LHCb, the Belle and the BaBar experiments. The excesses require the sizable couplings between the new physics and the SM. Then, we can expect that the new physics explanation is tested by the other processes not only in flavor physics but also in collider and DM physics. 


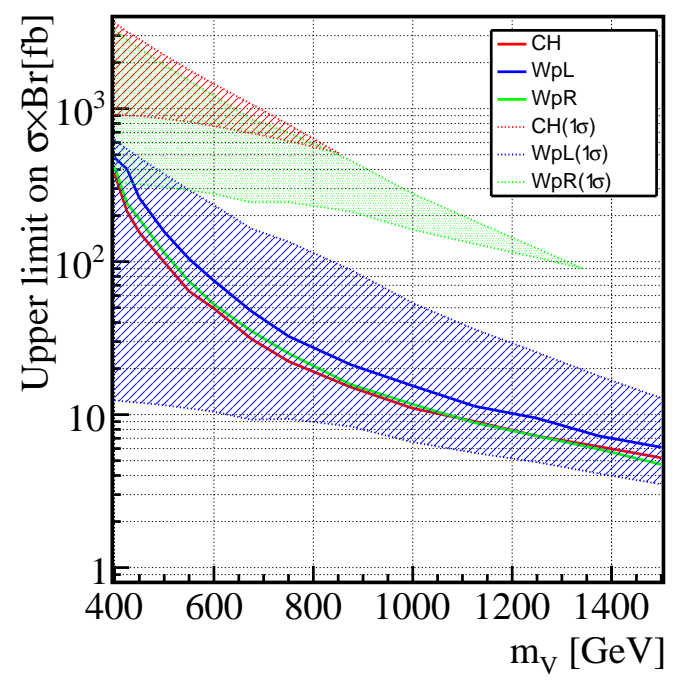

Figure 2: The upper bound on the cross section times branching ratio in the each model at 95\% CL [18].

The anomaly in the flavor experiment may be a hint to find out the physics beyond the SM. One possibility is that the dark sector is hidden behind the $b \rightarrow s l l$ anomaly. Another possibility is that new heavy resonance that couples to the SM fermions exists around the TeV scale. In both cases, we have seen that our models are almost excluded in a lot of parameter region, although our results may depend on our setup. We can find other models motivated by the excesses, e.g. leptoquark models $[20,26]$. In all cases, we can expect that the new physics interpretations can be tested by not only flavor physics but also the collider physics and the cosmological observation.

\section{References}

[1] R. Aaij et al. [LHCb Collaboration], Phys. Rev. Lett. 113, 151601 (2014) [arXiv:1406.6482 [hep-ex]].

[2] R. Aaij et al. [LHCb Collaboration], JHEP 1708, 055 (2017) [arXiv:1705.05802 [hep-ex]].

[3] R. Aaij et al. [LHCb Collaboration], JHEP 1509, 179 (2015) [arXiv:1506.08777 [hep-ex]].

[4] R. Aaij et al. [LHCb Collaboration], JHEP 1506, 115 (2015) [arXiv:1503.07138 [hep-ex]].

[5] R. Aaij et al. [LHCb Collaboration], Phys. Rev. Lett. 111, 191801 (2013) [arXiv:1308.1707 [hep-ex]].

[6] R. Aaij et al. [LHCb Collaboration], JHEP 1602, 104 (2016) [arXiv:1512.04442 [hep-ex]].

[7] J. P. Lees et al. [BaBar Collaboration], Phys. Rev. Lett. 109, 101802 (2012) [arXiv:1205.5442 [hep-ex]].

[8] J. P. Lees et al. [BaBar Collaboration], Phys. Rev. D 88, no. 7, 072012 (2013) [arXiv:1303.0571 [hep-ex]].

[9] M. Huschle et al. [Belle Collaboration], Phys. Rev. D 92, no. 7, 072014 (2015) [arXiv:1507.03233 [hep-ex]].

[10] Y. Sato et al. [Belle Collaboration], Phys. Rev. D 94, no. 7, 072007 (2016) [arXiv:1607.07923 [hep-ex]]. 
[11] S. Hirose et al. [Belle Collaboration], Phys. Rev. Lett. 118, no. 21, 211801 (2017) [arXiv:1612.00529 [hep-ex]].

[12] R. Aaij et al. [LHCb Collaboration], Phys. Rev. Lett. 115, no. 11, 111803 (2015) [arXiv:1506.08614 [hep-ex]].

[13] R. Aaij et al. [LHCb Collaboration], Phys. Rev. Lett. 120, no. 17, 171802 (2018) [arXiv:1708.08856 [hep-ex]].

[14] Talk by K. Adamczyk on " $B$ to semitauonic decays at Belle/Belle II" in $C K M$ 2018, Heidelberg, Germany, 17-21 September 2018.

[15] J. Kawamura, S. Okawa and Y. Omura, Phys. Rev. D 96, no. 7, 075041 (2017) [arXiv:1706.04344 [hep-ph]].

[16] P. Ko, Y. Omura, Y. Shigekami and C. Yu, Phys. Rev. D 95, no. 11, 115040 (2017) [arXiv:1702.08666 [hep-ph]].

[17] P. Ko, Y. Omura and C. Yu, JHEP 1303, 151 (2013) [arXiv:1212.4607 [hep-ph]].

[18] S. Iguro, Y. Omura and M. Takeuchi, Phys. Rev. D 99, no. 7, 075013 (2019) [arXiv:1810.05843 [hep-ph]].

[19] W. Altmannshofer, P. Stangl and D. M. Straub, Phys. Rev. D 96, no. 5, 055008 (2017) [arXiv:1704.05435 [hep-ph]].

[20] D. Buttazzo, A. Greljo, G. Isidori and D. Marzocca, JHEP 1711, 044 (2017) [arXiv:1706.07808 [hep-ph]].

[21] T. Abe, J. Kawamura, S. Okawa and Y. Omura, JHEP 1703, 058 (2017) [arXiv:1612.01643 [hep-ph]].

[22] D. S. Akerib et al. [LUX Collaboration], Phys. Rev. Lett. 116, no. 16, 161301 (2016) [arXiv:1512.03506 [astro-ph.CO]].

[23] D. S. Akerib et al., arXiv:1608.07648 [astro-ph.CO].

[24] E. Aprile et al. [XENON Collaboration], arXiv:1705.06655 [astro-ph.CO].

[25] A. M. Sirunyan et al. [CMS Collaboration], [arXiv:1807.11421 [hep-ex]].

[26] S. Iguro, T. Kitahara, Y. Omura, R. Watanabe and K. Yamamoto, JHEP 1902, 194 (2019) [arXiv:1811.08899 [hep-ph]]. 